\title{
Machine Learning Algorithmic Study of the Naira Exchange Rate
}

\author{
Ledisi G. Kabari, Marcus B. Chigoziri, and Eneotu Joseph
}

\begin{abstract}
In this study, we discuss various machine learning algorithms and architectures suitable for the Nigerian Naira exchange rate forecast. Our analyses were focused on the exchange rates of the British Pounds, US Dollars and the Euro against the Naira. The exchange rate data was sourced from the Central Bank of Nigeria. The performances of the algorithms were evaluated using Mean Squared Error, Root Mean Squared Error, Mean Absolute Error and the coefficient of determination (R-Squared score). Finally, we compared the performances of these algorithms in forecasting the exchange rates.
\end{abstract}

Index Terms-Exchange Rate, Machine Learning, Recurrent Neural Network, Time Series Forecast.

\section{INTRODUCTION}

The importance of exchange rate forecast cannot be overemphasized. It is believed that exchange rate volatility hampers the growth of international trade [1]. The effects of exchange rate variability [2] have been a subject of discuss in international economics [3], [4]. Exchange rate forecast help businesses to evaluate risks and make proactive decisions in order to maximize returns. In the 1970's, the introduction of floating exchange rates led to a dynamic change in the international financial market. Since then, exchange rates have been determined largely by private market forces within the floating exchange rate system. In a densely populated country like Nigeria, which is a high target for international investments, import and even export of goods, it is imperative to study foreign currency exchange rates.

In this paper, we obtained, observed and analyze the history of major foreign currencies exchange rates in the world against the naira in simple time series plots. We used various machine learning algorithms such as the support vector machines, linear and polynomial regressions and recurrent neural network to train the historic data and forecast the next future exchange rates. We implemented the algorithms in Python programming language using the TensorFlow and Scikit-Learn (sklearn) frameworks, and MATLAB. We also used NumPy and Pandas libraries for data preprocessing. The simulation was done using the daily historic exchange rate data of a period of over 18 years,

Published on February 17, 2020.

L. G. Kabari is with Computer Science Department, Ken Saro-Wiwa Polytechnic, Bori, Nigeria (e-mail: ledisigiokkabari@ yahoo.com).

M. B. Chigoziri is a Post Graduate Student of Computer Science Department, Ignatius Ajuru University of Education, Port Harcourt, Nigeria (e-mail: chigozirimarcus@yahoo.com).

J. Eneotu is a Post Graduate Student of Computer Science Department, Ignatius Ajuru University of Education, Port Harcourt, Nigeria (eneotujoe@gmail.com). obtained from the Central Bank of Nigeria [5]. Fig. 1 represents a plot of the yearly average exchange rates of the US Dollar (USD), British Pounds (GBP) and Euro (EUR) to the Nigerian Naira (NGN).

The remaining parts of this paper are structured as follows: The related works are in Section II, Section III presents the different machine learning algorithms used, the methodology is presented in Section IV, results and discussions are in Section V and finally, the conclusion is in Section VI.

\section{RELATED WORKS}

Reference [6] carried out a research on predicting the exchange rates of the US Dollar, British Pounds, Japanese YEN and other currencies, to the Nigerian Naira. They used Multiple Linear Regression and MATLAB to perform the analysis. Reference [7] applied Seasonal Arima Model in forecasting the exchange of the naira. In the context the Nigerian naira, these references never applied machine learning algorithms to analyze and compare their findings. Our primary focus on this is to apply different machine learning algorithms, including the state-of-the-art Deep Neural Network (DNN) in foretelling the exchange rate of foreign currencies against the Nigerian naira. Existing literature shows the use of Auto-Regressive conditional Heteroskedasticity (ARCH) [8] and Generalized AutoRegressive conditional Heteroskedasticity (GARCH) [9] models for forecasting stock prices and other time series data predictions. In these days of cryptocurrencies (particularly bit- coin) that is disrupting the financing institutions, reference [10] points out that Long-Short Term Memory (a type of Recurrent Neural Network) is more successful in making prediction on bitcoin time series data. References [11],[12],[13] and others have used recurrent neural network in predicting the price of bitcoin. However, our work and interest lies in predicting the exchange rates of foreign currencies to the NGN.

\section{MATH}

In this section, the machine learning algorithms used in this paper are presented.

\section{A. Polynomial Regression}

In the context of machine learning, polynomial regression can be transformed into linear regression. The general mathematical equation for a polynomial of $\mathrm{n}^{\text {th }}$ degree is:

$$
y(w, x)=w_{0}+w_{1} x+w_{2} x^{2}+\ldots+w_{n} x^{n}
$$




\section{Where $x=x_{1}+x_{2}+\ldots+x_{n}$}

$$
\text { and } w=w_{1}+w_{2}+\ldots+w_{n}
$$

A second-order (quadratic) polynomial can be written as: [14]

$$
y(w, x)=w_{2} x^{2}+w_{1} x+w_{0}
$$

Which can be reformulated as:

$y(w, x)=w_{5} x_{2}^{2}+w_{4} x_{1}^{2}+w_{3} x_{1} x_{2}+w_{2} x_{2}+w_{1} x_{1}+w_{0}(3)$

Let $\quad$ say $\quad z=\left[x_{2}^{2}, x_{1}^{2}, x_{1} x_{2}, x_{1}, x_{2}\right]=\left[z_{5}, z_{4}, z_{3}, z_{2}, z_{1}\right]$ Therefore,

$y(w, x)=w_{5} z_{5}+w_{4} z_{4}+w_{3} z_{3}+w_{2} z_{2}+w_{1} z_{1}+w_{0}$

This shows that the polynomial equation (2) has been transformed into a linear equation (4) in $w$. In this way, a polynomial regression problem can be remodeled as a linear one. In other words, polynomial curves can be used as a sort of replacement for transformations of linear functions. Though they tend to be more useful in situations where residual variation is small, they have occasional uses. Moreover, they provide a simple setting for considering the variable selection problem. To apply a polynomial in multiple linear regression model, one merely constructs a set of variables which are powers of the desired base variable.

For the purpose of this research, the sklearn polynomial feature api [15] and the polyval/polyfit tools in MATLAB were used and the results were compared.

\section{B. Support Vector Machines}

Support Vector Machines (SVMs), also known as Support Vector Networks are a machine learning algorithm for classification and regression problems [16]. SVM is an algorithm that maps input data into a high-dimensional space and it uses the concept of hyper-plane to maximally separate training data. Given the constraint,

$$
\begin{aligned}
& y_{i}\left(w x_{i}+b\right) \geq 1 \\
& \text { where } i=1, \ldots l
\end{aligned}
$$

For higher generalization of the learning architecture, the optimal hyper-plane, given by:

$$
w_{0} \cdot x+b_{0}=0
$$

is used. The optimal hyper-plane passes through the maximum margin between the support vectors of the classes in the dataset. [16].

The support vectors $x_{i}$ are obtained from the equation:

$$
y_{i}\left(w \cdot x_{i}+b\right)=1
$$

Support Vector Classification (SVC) is used for classification problems whereas Support Vector Regression (SVR) is used for regression and for this paper, we will use SVR. In this work, we used the sklearn library to implement the SVR with rbf, linear and polynomial kernels.

\section{Neural Networks}

A Neural Network (NN) is a set of computational algorithms inspired by the neurons in the brain. A simple NN also known as the single-layer perceptron, can be described as a function $f$ which maps input data $x$ to an output vector $y$ as shown in equation 8 .

$$
y=f(w \cdot x+b)
$$

where $w$ is the weight, and $b$ is the bias. In this section, we shall be considering a popularly used $\mathrm{NN}$ algorithms.

\section{Recurrent Neural Networks}

A Recurrent Neural Net- work (RNN) is a type of NN that is designed to learn from sequence of data. The basic problem of RNN is the vanishing gradient problem, however, this is solved by the Long-Short Term Memory (LSTM) architecture, a type of RNN introduced by Hochreiter et al [17]. In this paper we implemented the Gated Recurrent Unit (GRU), which is another variant of the RNN, and also a combined implementation of GRU and LSTM.

\section{Methodology}

\section{A. Data}

The data for this research was obtained from the Central Bank of Nigeria $(\mathrm{CBN})$. The data contain the exchange rates of 9 foreign currencies which include USD, GBP, KRONER, YEN, EUR and other currencies, from December 2001 to September 2019. Our research is based on the exchange rates of British Pounds Sterling, United States Dollar and the Euro against the Nigerian Naira (NGN). However, for training the machine learning algorithms, we used the USD. The data contains 7 columns with buying, selling and central (average) exchange rates, and 41583 rows of samples. Fig. 1 represents a plot of the data resampled to yearly average exchange rates.

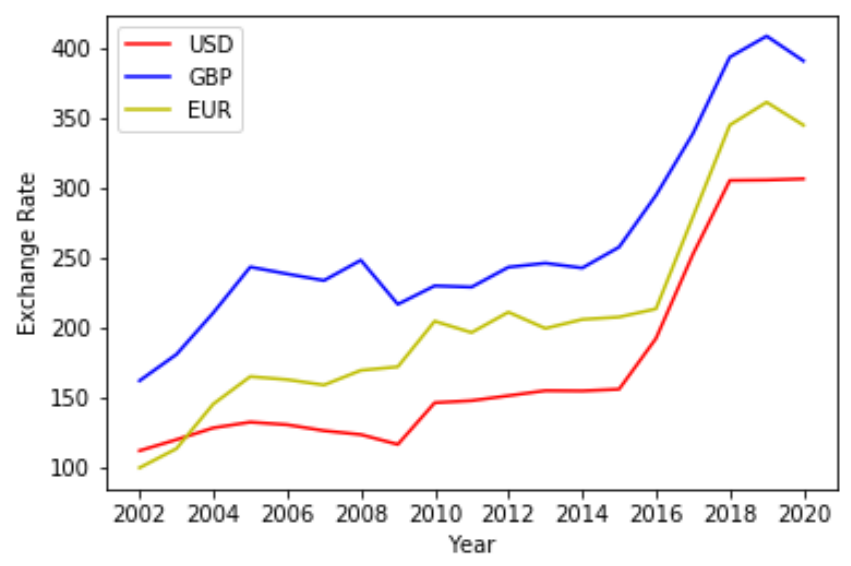

Fig. 1. Yearly Average Exchange Rates of USD/NGN, GBP/NGN and EUR/NGN

\section{B. Data Preprocessing and Normalization}

The most important aspect of using data for training any machine learning algorithm is data preprocessing. Here we preprocessed the data using NumPy and Pandas libraries. We called different functions on Pandas Dataframe of the data to check and remove missing and none numeric values 
before converting the data into NumPy array. The data was normalized using sklearn MinMaxScaler function and traintest-split function to split the data into $80 \%$ train set and $20 \%$ for validation before inputting into the learning algorithms.

\section{Hyperparameter, Activation Functions and Overfitting}

Hyperparameter tuning is one of the salient techniques when training a neural network. Dropout, activation function, optimizer and loss function are the hyparameters that we fine-tuned to obtain a better result. We used the relu activation function, Adam optimizer, Mean Absolute Error loss function and a dropout of 0.4 to train the neural networks. Overfitting is a situation where the network learns the training set better with lower training losses but when the trained model is tested with unseen data or the validation set, it does not generalize properly. The purpose of the dropout in this paper is to reduce overfitting.

\section{Evaluation Metrics}

For this research, we used Mean Squared Error (MSE), Root Mean Squared Error (RMSE), Mean Absolute Error (MAE) and the R-Squared $\left(R^{2}\right)$ score metrics. Given a dataset with the $i^{\text {th }}$ true label as $y$ and $\hat{y}$ as the corresponding predicted ii label, the MSE, RMSE, MAE and the $R^{2}$ can be estimated by using equations (9), (10), (11) and (12) respectively below, with $n$ as the number of samples.

$$
\begin{aligned}
& \operatorname{MSE}\left(y_{i}, \hat{y_{i}}\right)=\frac{1}{n} \sum_{i=1}^{n}\left(y_{i}-\hat{y_{i}}\right)^{2} \\
& \operatorname{RMSE}\left(\hat{y}_{i}, \hat{y_{i}}\right)=\sqrt{\frac{1}{n} \sum_{i=1}^{n}\left(y_{i}-\hat{y_{i}}\right)^{2}} \\
& \operatorname{MAE}\left(y_{i}, \hat{y_{i}}\right)=\frac{1}{n} \sum_{i=1}^{n}\left|y_{i}-\hat{y_{i}}\right| \\
& R^{2}\left(y_{i}, \hat{y}_{i}\right)=1-\frac{\sum_{i=1}^{n}\left(y_{i}-\hat{y}_{i}\right)^{2}}{\sum_{i=1}^{n}\left(y_{i}-\overline{y_{i}}\right)^{2}}
\end{aligned}
$$

Where $\overline{y_{i}}=\frac{1}{n} \sum_{i=1}^{n} y_{i}$

\section{E. Implementation}

Finally, we implemented the RNNs (GRU and LSTM) algorithms using TensorFlow. The SVR and its various kernels were implemented in sklearn. We also implemented the polynomial regression in MATLAB. Then we used Matplotlib library to visualize every relevant information in the training and validation phases. The interesting results are presented in Section V.

\section{RESULTS AND DISCUSSION}

In this section, we present the results and performances of the classical machine learning as well as the deep learning algorithms.

Starting with the polynomial regression, Fig. 2 is the graph showing the validation of the polynomial regression algorithm. Using the degree argument of the polynomial features of 2 (degree $=2$ ), we obtained the validation performance of the polynomial regression algorithm as: $\mathrm{MSE}=0.0017, \mathrm{RMSE}=0.0407, \mathrm{MAE}=0.0050$ and $\mathrm{R}$ Squared score $=0.9677$. In MATLAB, the computed annual averages of the exchange rates were used to forecast the GBP and EUR for 2020 and 2022 as shown in Table I.

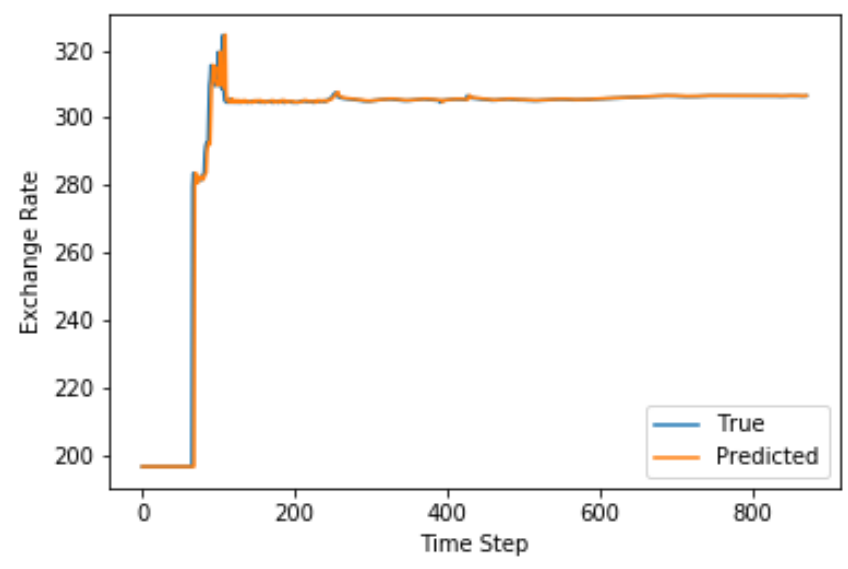

Fig. 2. Polynomial Regression

TABLE I: PROJECTION OF GBP AND EUR IN MATLAB

\begin{tabular}{lll}
\hline \hline Currency & 2020 & 2022 \\
\hline GBP & 372.9237 & 394.6418 \\
\hline EUR & 330.7031 & 355.0134 \\
\hline \hline
\end{tabular}

In Fig. 3, we present the graphs of rbf kernel of the Support Vector Regression algorithm, though we also implemented the linear and polynomial kernels of the SVR. The performances of the 3 SVR kernels are shown on Table II. The performance of SVR polynomial kernel and the actual polynomial regression above in Fig. 2 differs a little. This should be due to the tweaking of some argument variables in the regression.

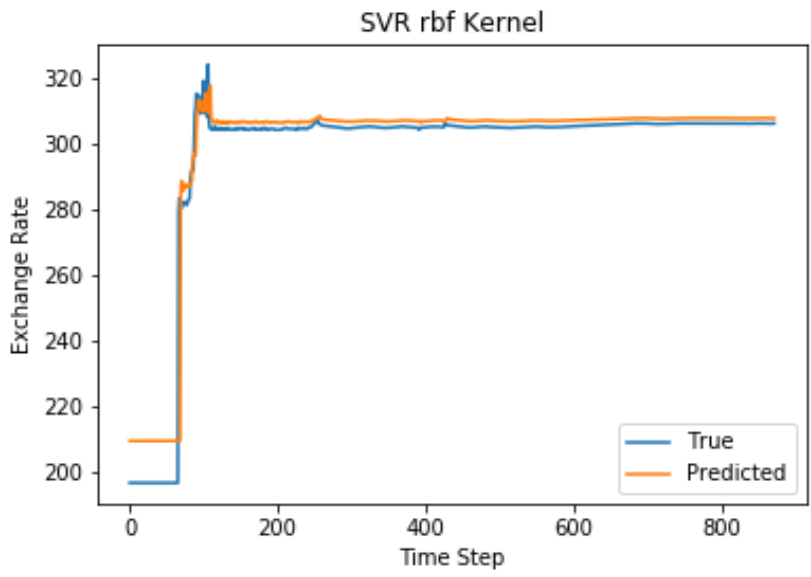

Fig. 3. SVR with rbf kernel

TABLE II: SVR ERROR AND CONFIDENCE

\begin{tabular}{ccccc}
\hline \hline SVR Kernel & MSE & RMSE & MAE & $\mathrm{R}^{2}$ \\
\hline rbf & 0.00214 & 0.04621 & 0.02367 & 0.958 \\
\hline Linear & 0.00181 & 0.04255 & 0.01841 & 0.965 \\
\hline Polynomial & 0.00331 & 0.05755 & 0.02288 & 0.935 \\
\hline \hline
\end{tabular}


We trained the GRU algorithm with mean absolute error loss for 10 epochs and the result is stunning. Fig. 5 is the plot of the loss on every epoch of training, and Fig. 4 shows the exchange rate plot from the validation set. Table III shows the MAE, MSE and RMSE losses on both training and testing. Similar performance was obtained on the LSTM/GRU combined architecture. For LSTM/GRU, we obtained the MAE, MSE and RMSE on the test set to be $0.008710,0.000317$ and 0.017805 respectively.

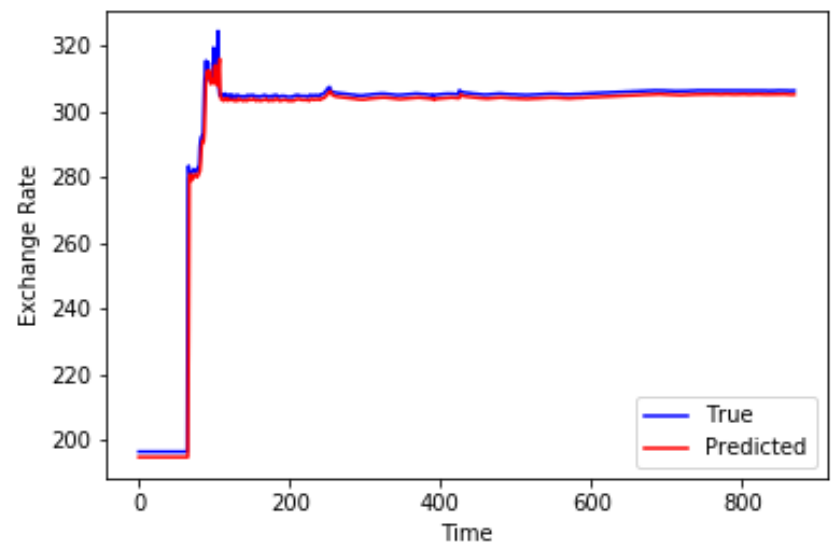

Fig. 4. The Gated Recurrent Unit

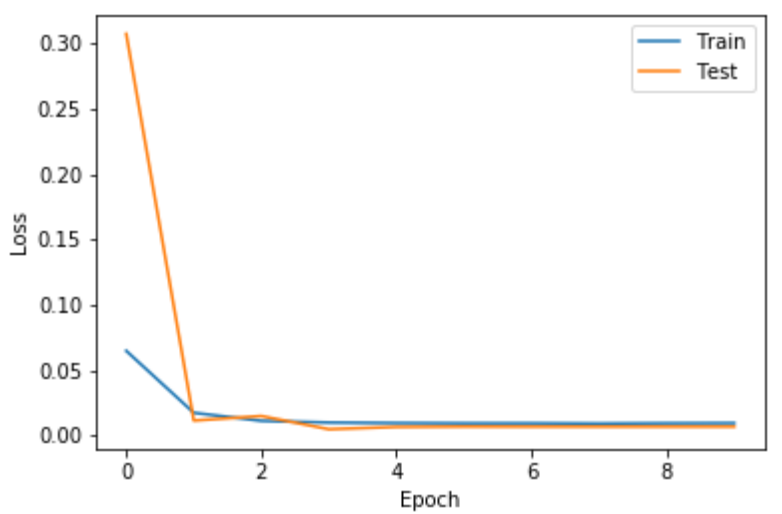

Fig. 5. GRU Graph of Training Losses against Epochs

TABLE III: GRU TRAINING AND VALIDATION LOSSES

\begin{tabular}{cccc}
\hline \hline Metrics & Loss (MAE) & MSE & RMSE \\
\hline Training Loss & 0.003698 & 0.000029 & 0.005423 \\
\hline Validation Loss & 0.007070 & 0.000318 & 0.017833 \\
\hline \hline
\end{tabular}

\section{CONCLUSION}

In this paper, we experimented the forecast of the foreign currency to naira exchange rate with various machine learning algorithms. We evaluated the performance of these algorithms and obtained interesting results which show that the Recurrent Neural Networks are more successful in time series analysis and forecast. The exchange rates considered in this work are daily exchange of the US Dollar to the naira. We also took annual average of the exchange rates and used MATLAB polyfit/polyval to obtain the 2020 and 2022 projections of the GBP and EUR to NGN exchange rates.

\section{REFERENCES}

[1] I. Ozturk, "Exchange rate volatility and trade: A literature survey," International Journal of Applied Econometrics and Quantitative Studies, vol. 3, no. 1, 2006.

[2] T. Caporale and K. Doroodian, "Exchange rate variability and the flow of international trade," Economics Letters, vol. 46, no. 1, pp. 4954, 1994.

[3] B. Coric' and G. Pugh, "The effects of exchange rate variability on international trade: a meta-regression analysis," Applied Economics, vol. 42, no. 20, pp. 2631-2644, 2010.

[4] M. J. Bailey, G. S. Tavlas, and M. Ulan, "Exchange-rate variability and trade performance: evidence for the big seven industrial countries," Review of World Economics, vol. 122, no. 3, pp. 466-477 1986.

[5] Central Bank of Nigeria, "Cbn exchange rates," September 2019. [Online].

Available: https://www.cbn.gov.ng/rates/ExchRateByCurrency.

[6] L. Kabari and B. Nwamae, "Stochastic analysis of the exchange rate of naira, yen, gbp, cfa and franc in relation to us dollar and predicting the naira for the year 2025," European Journal of Engineering Research and Science, vol. 4, no. 6, pp. 15-18, Jun. 2019. [Online]. Available: https://www.ejers.org/index.php/ejers/article/view/1353

[7] E. H. Etuk, "Forecasting nigerian naira-us dollar exchange rates by a seasonal arima model," American Journal of scientific research, vol. 59, pp. 71-78, 2012.

[8] R. F. Engle, "Autoregressive conditional heteroscedasticity with estimates of the variance of United Kingdom inflation," Econometrica: Journal of the Econometric Society, pp. 987-1007, 1982.

[9] T. Bollerslev, "Generalized autoregressive conditional heteroskedasticity," Journal of econometrics, vol. 31, no. 3, pp. 307 327, 1986.

[10] E. Karakoyun and A. Cibikdiken, "Comparison of arima time series model and lstm deep learning algorithm for bitcoin price forecasting," in The 13th Multidisciplinary Academic Conference in Prague 2018 (The 13th MAC 2018), 2018, pp. 171-180.

[11] S. McNally, J. Roche, and S. Caton, "Predicting the price of bitcoin using machine learning," in 2018 26th Euromicro International Conference on Parallel, Distributed and Network-based Processing (PDP). IEEE, 2018, pp. 339-343.

[12] D. R. Pant, P. Neupane, A. Poudel, A. K. Pokhrel, and B. K. Lama, "Recurrent neural network based bitcoin price prediction by twitter sentiment analysis," in 2018 IEEE 3rd International Conference on Computing, Communication and Security (ICCCS). IEEE, 2018, pp. 128-132.

[13] S. Lahmiri and S. Bekiros, "Cryptocurrency forecasting with deep learning chaotic neural networks," Chaos, Solitons \& Fractals, vol. 118, pp. 35-40, 2019.

[14] F. Pedregosa, G. Varoquaux, A. Gramfort, V. Michel, B. Thirion, O. Grisel, M. Blondel, P. Prettenhofer, R. Weiss, V. Dubourg, J. Vanderplas, A. Passos, D. Cournapeau, M. Brucher, M. Perrot, and E. Duchesnay, "Scikit-learn: Machine learning in Python," Journal of Machine Learning Research, vol. 12, pp. 2825-2830, 2011.

[15] L. Buitinck, G.Louppe, M. Blondel, F. Pedregosa, A. Mueller, O. Grisel, V. Niculae, P. Prettenhofer, A. Gramfort, J. Grobler, R. Layton, J. Vander Plas, A. Joly, B. Holt, and G. Varoquaux, "API design for machine learning software: experiences from the scikitlearn project," in ECML PKDD Workshop: Languages for Data Mining and Machine Learning, 2013, pp. 108-122.

[16] C. Cortes and V. Vapnik, "Support-vector networks," Machine learning, vol. 20, no. 3, pp. 273-297, 1995.

[17] S. Hochreiter and J. Schmidhuber, "Long short-term memory," Neural computation, vol. 9, no. 8, pp. 1735-1780, 1997. 